\title{
Floreallndices: Une feuille de calcul pour produire des indices agroécologiques de prairies permanentes à partir du relevé de végétation
}

\author{
Jean-Pierre Theau ${ }^{1}(1)$ et François Prud'homme, ${ }^{2, *}$ \\ 1 INRAE, UMR 1248 AGIR, F-31326 Castanet-Tolosan, France \\ 2 Conservatoire botanique national des Pyrénées et de Midi-Pyrénées, Syndicat mixte Conservatoire botanique pyrénéen, Vallon de Salut, \\ BP 315, F-65203 Bagnères-de-Bigorre Cedex, France
}

\begin{abstract}
Résumé - Le Conservatoire botanique national des Pyrénées et de Midi-Pyrénées (CBNMP) et l'Institut national de recherche pour l'agriculture, l'alimentation et l'environnement de Toulouse (INRAE) ont produit en accès libre une base de données des 768 espèces prairiales de Midi-Pyrénées avec leurs attributs : FlorealData (https://doi.org/10.15454/ADCQHT). Nous présentons ici une feuille de calcul «FlorealIndices», qui permet, en utilisant le relevé phytosociologique de la station, de calculer automatiquement une dizaine d'indices agronomiques et écologiques. La production de ces indices permet aux botanistes, qu'ils soient agronomes ou plus généralement écologues, de s'approprier des concepts issus de ces deux disciplines afin d'en faciliter l'hybridation.
\end{abstract}

Mots clés : agroécologie / data paper / prairie permanente / calcul d'indices / agronomie / phytosociologie / gestion

\begin{abstract}
Floreallndices: A spreadsheet to produce agroecological indices of permanent grasslands from the vegetation survey. The National Botanical Conservatory of the Pyrénées and Midi-Pyrénées (CBNMP) and the National Research Institute for Agriculture, Food and Environment of Toulouse (INRAE) have produced a free access database of 768 meadow species of Midi-Pyrénées with their attributes: FlorealData (https://doi.org/10.15454/ADCQHT). We present here a spreadsheet "FlorealIndices", which allows, using the plant phytosociological record, to automatically calculate around ten agronomic and ecological indices. The production of these indices allows botanists, whether they are agronomists or more generally ecologists, to appropriate concepts from these two disciplines in order to facilitate hybridization.
\end{abstract}

Keywords: agroecology / data paper / permanent grassland / index calculation / agronomy / phytosociology / management

\section{Contexte scientifique}

Les prairies permanentes, ressources essentielles des grandes zones herbagères de montagne, sont aujourd'hui mises en avant au travers de nombreuses typologies prairiales qui établissent des liens entre leur diversité botanique et les services écosystémiques qu'elles produisent (Plantureux et al., 2012 ; Carrère et al., 2012; Galliot et al., 2019). En plaine, leur maintien ou leur essor se heurte encore à une image passéiste face à des modèles de production intensifs. Pourtant, leur intégration comme élément paysager, au même titre que la forêt ou les haies, dans des espaces agricoles en fait une

\footnotetext{
*Auteur de correspondance : francois.prudhomme@cbnpmp.fr
}

ressource essentielle dans la construction de systèmes agroécologiques (Theau et Choisis, 2016). Ainsi, leur érosion entraîne un maillage de plus en plus diffus en plaine, qui nuit aux nombreux services écosystémiques que la prairie permanente procure. Les deux communautés, d'agronomes et de phytosociologues, qui s'intéressent à la prairie permanente ont des objectifs d'étude différents (productivité des systèmes vs description, conservation des habitats et des espèces) et de fait mobilisent des concepts, des méthodes et des niveaux d'échelle différents. Pourtant, les deux communautés se retrouvent autour de la nécessaire prise en compte des pratiques agricoles dans la pérennité des prairies permanentes. Ce point de vue est conforté par un renforcement récent des conventions de l'Institut national de recherche pour l'agriculture, l'alimentation et l'environnement(INRAE) et des 
Tableau 1. Le pourcentage de recouvrement de l'espèce utilise la médiane des classes d'abondance dominance de Braun-Blanquet. Cette transformation faite, il faut normaliser à 1 la somme des recouvrements d'espèces pour chacune des stations et importer le bloc de données espèces stations, dans la cellule M1 de la feuille de calcul.

Table 1. The species recovery percentage uses the median of the Braun-Blanquet dominance abundance classes. Once this transformation is done, we must normalize the sum of the cash recoveries for each of the stations to 1 and import the station species data block, into cell M1 of the spreadsheet.

\begin{tabular}{lcccccc}
\hline Abondance dominance (Braun-Blanquet, 1964) & 5 & 4 & 3 & 2 & 1 & + \\
Pourcentage de recouvrement (Dufrêne, 1998) & 87,5 & 62,5 & 37,5 & 15 & 2,5 & 0,2 \\
\hline
\end{tabular}

Conservatoires botaniques nationaux $(\mathrm{CBN})$ avec l'Office français de la biodiversité, fortement préoccupé par l'érosion de la biodiversité terrestre et notamment celle des prairies permanentes. Au-delà de la reconnaissance des espèces, un des freins à l'articulation des travaux de ces deux disciplines réside dans la différence de référentiels. Les référentiels taxonomiques tout d'abord: ils sont précisés dans les travaux phytosociologiques qui servent de base aux politiques publiques de conservation des habitats, mais sont très peu présents dans les travaux agronomiques. Les référentiels typologiques ensuite : nous avons montré, sur un échantillon de 435 prairies permanentes de Midi-Pyrénées, que le croisement de deux typologies de prairies, l'une phytosociologique, l'autre agronomique, donnait lieu à de multiples intersections aux caractéristiques agroécologiques parfois très variables (Theau et al., 2019). Or, si l'on veut conserver et pérenniser des habitats particuliers au travers de pratiques agricoles, il est nécessaire pour les gestionnaires d'espaces naturels de mieux les décrire objectivement au travers d'indices, à partir du relevé botanique. C'est ce que nous avons développé depuis plusieurs années en Midi-Pyrénées (Prud'homme et Theau, 2017, 2019; Theau et al., 2019) et que nous proposons de structurer dans ce papier en rendant accessible leur calcul. Ces indices permettent de croiser des points de vue d'agronomes et d'écologues botanistes afin de les hybrider dans une écoute mutuelle. Ils nous semblent indispensables pour éclairer des discussions objectives avec l'éleveur, qui au final est celui qui entretient ces milieux par ses pratiques fourragères.

\section{Méthodologie}

\subsection{La feuille de calcul Floreallndices}

Cette feuille de calcul, développée dans Excel, permet de calculer automatiquement des indices agroécologiques à partir d'un relevé botanique exhaustif sur une station homogène (relevé phytosociologique). FlorealIndices est mis à disposition sous Licence Ouverte (Licence CCO Transfert dans le domaine public), autorisant toute utilisation sous réserve de citer les auteurs. Afin de lui donner de la visibilité auprès de la communauté scientifique, les données sont exposées et téléchargeables sur le portail des données institutionnel Data INRAE (Data INRAE, 2019), qui permet l'obtention d'un Digital Object Identifier (DOI : https://doi.org/10.15454/ ADCQHT). La feuille est structurée en trois zones :

- une zone d'importation (M810) de la base de données FlorealData regroupant les principales espèces prairiales de Midi-Pyrénées (Theau et Prud'homme, 2021). Ce fichier sous licence ouverte est à télécharger sur le même portail de données Data INRAE;
- une zone d'importation des relevés botaniques (M1);

- une zone de calcul et de restitution des indices (M786 à M800).

Un fichier «lisez moi.pdf» est disponible afin de guider l'utilisateur de FlorealIndices.

\subsection{Préparation des données du relevé botanique}

\subsubsection{Transformation de l'abondance dominance des} espèces en pourcentage de recouvrement

Les espèces du relevé phytosociologique (en ligne) doivent être nommées suivant le référentiel Taxref 12 pour chacune des stations (en colonnes). À l'intersection des lignes et des colonnes, l'abondance/dominance selon la méthode BraunBlanquet (1964) a été transformée (Tab. 1) en pourcentage de recouvrement (Dufrêne, 1998). Il est possible d'utiliser une autre méthode d'estimation de l'abondance des espèces, dès lors que la somme des abondances est égale à 1 pour chacune des stations. Nous attirons cependant l'attention des utilisateurs sur le biais que cela introduit, si l'on souhaite comparer les résultats avec notre base de données (Fig. 1). Selon les indices, les calculs se font sur la base de la seule présence/ absence des espèces ou intègrent une pondération reflétant leur abondance dans la station.

\subsubsection{Récupération des traits biologiques de FlorealData pour les espèces du relevé}

Une fonction RechercheV d'Excel va identifier automatiquement si l'espèce trouvée dans le relevé est bien référencée dans FlorealData. Si oui, le nom apparaît et la feuille de calcul importe dans les colonnes A à J les attributs de l'espèce. Si non, \#N/A apparaît dans la colonne K, qui signifie que l'espèce est absente de FlorealData. La colonne L effectue un calcul de recouvrement relatif de l'espèce dans le dispositif, afin de voir s'il y a lieu de compléter ces informations dès lors que l'espèce apparaît dominante. Si c'est le cas, la méthodologie de recherche des attributs est décrite dans FlorealData (Theau et Prud'homme, 2021). Les \#N/A et les 0 interférant avec certains calculs d'indices, il est nécessaire de les supprimer dans les colonnes $\mathrm{A}$ à $\mathrm{J}$ avant tout calcul (attention, il peut y avoir des valeurs proches de 0 pour le SSI qu'il faut conserver). Ces différentes étapes sont expliquées dans le «lisez moi.pdf» qui accompagne FlorealIndices. L'automatisation de ces quelques étapes est en cours pour les développements futurs de l'outil.

Enfin, la ligne 772 de FlorealIndices permet de voir si le cumul d'abondances des espèces de la parcelle est bien renseigné par FlorealData. Nous considérons qu'en 


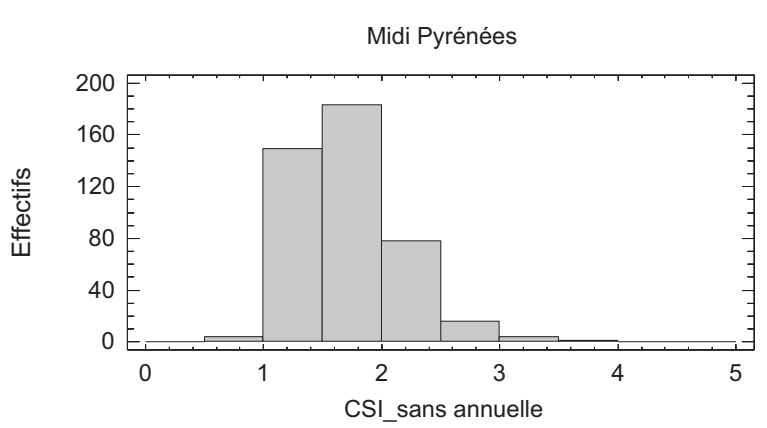

Midi Pyrénées
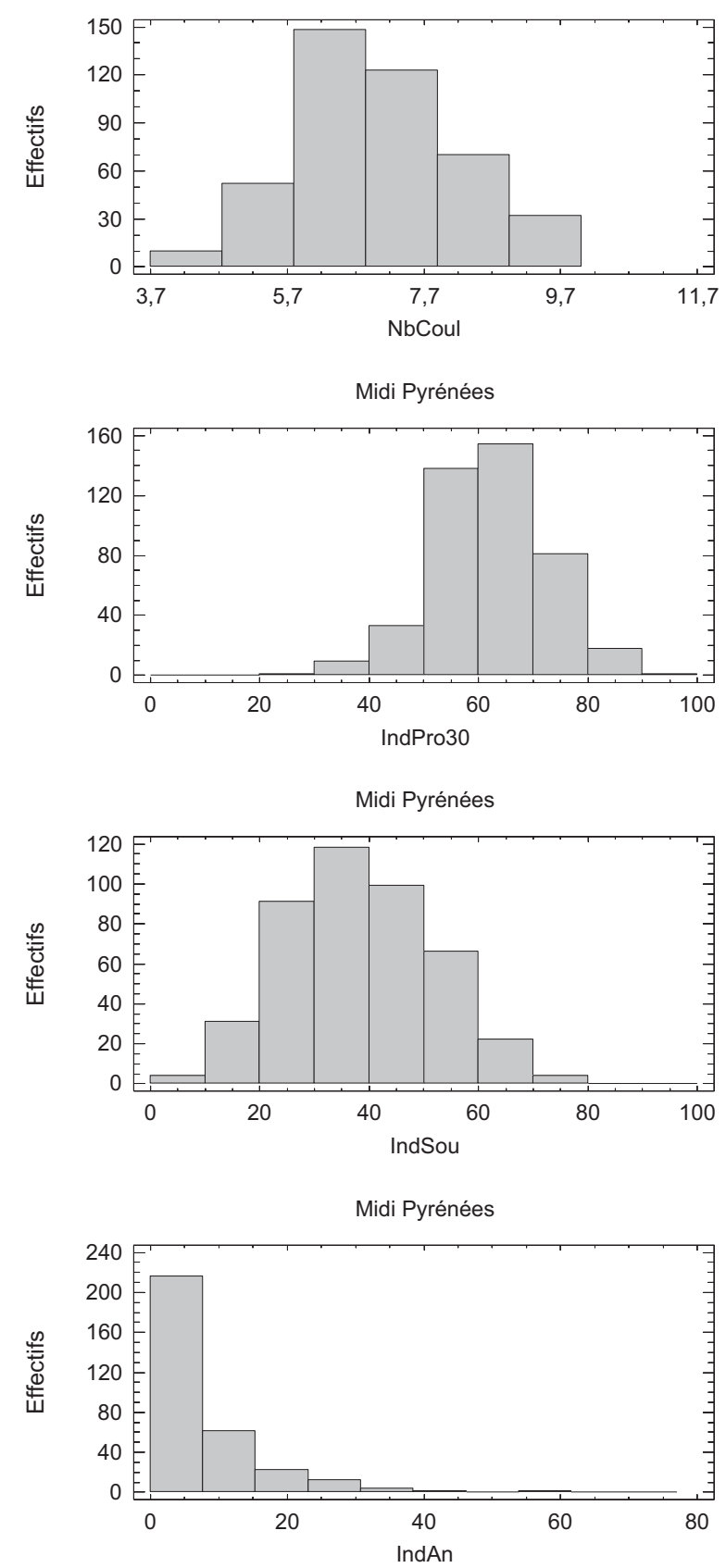
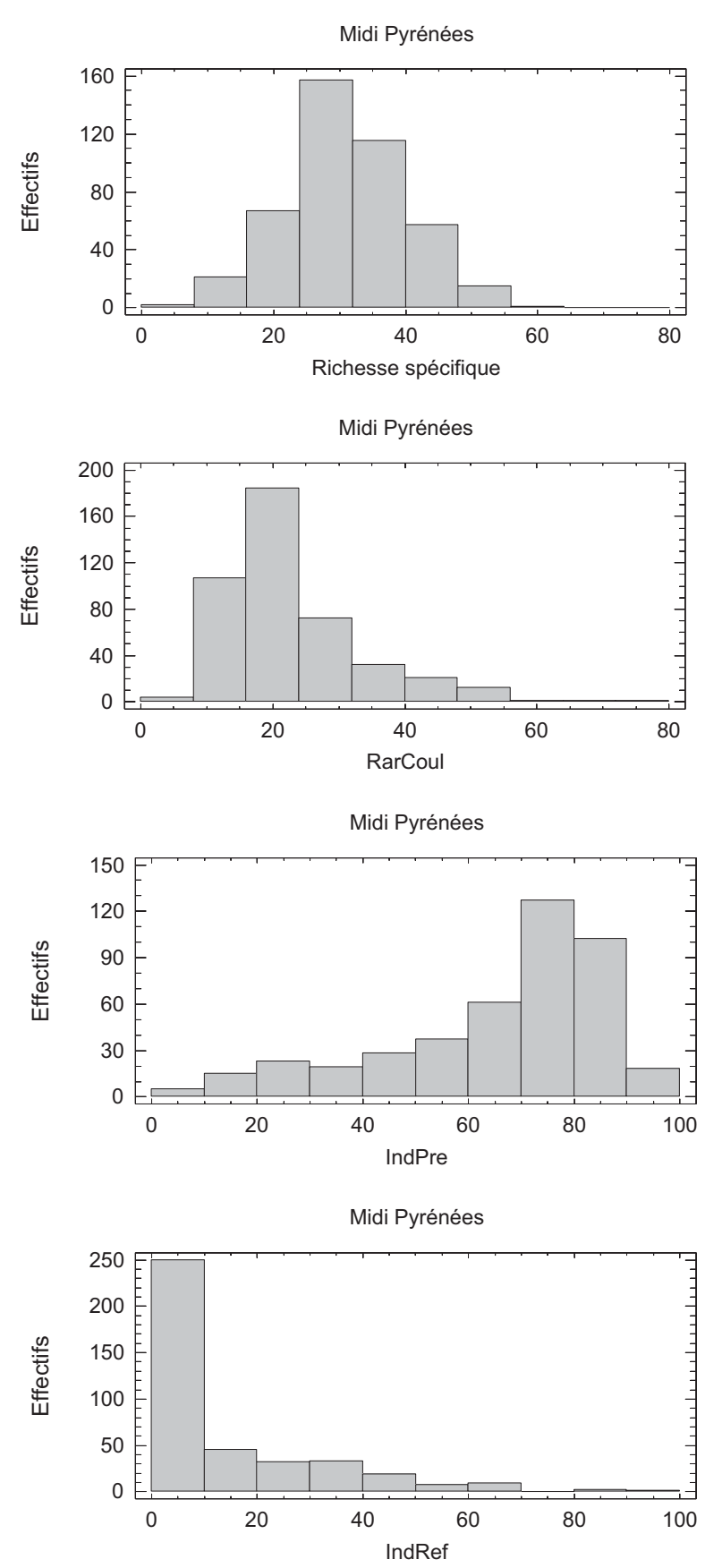

Fig. 1. Histogrammes de fréquences de 435 prairies permanentes de Midi-Pyrénées pour neuf indices agroécologiques calculés par FlorealIndices.

Fig. 1. Frequency histograms of 435 permanent meadows in Midi-Pyrénées for nine agroecological indices calculated by FlorealIndices. 
dessous de $80 \%$ d'abondance, la validité des indices est compromise.

FlorealData a été produit sur le territoire de Midi-Pyrénées, sous influences principalement atlantiques et montagnardes. Son utilisation avec FlorealIndices a donc une limite de validité biogéographique. En effet, si les indices sont génériques, certains traits pourraient avoir des valeurs différentes dans d'autres domaines biogéographiques, en particulier dans le domaine méditerranéen.

\subsection{Indexation de chaque espèce à un Type fonctionnel de plante (TFP)}

Dans FlorealData, chaque espèce est indexée par un TFP. Celui-ci est renseigné pour les graminées par la typologie fonctionnelle des graminées établies par Cruz et al. (2010). Pour les autres espèces (Légumineuses, Cypéracées, Juncacées et autres), nous nous sommes inspirés de Theau et al. (2017) en combinant la hauteur moyenne de l'espèce et sa précocité de floraison. Le tableau 2 présente les 7 types fonctionnels de graminées et 36 types fonctionnels de non-graminées ainsi que leur niveau de contribution aux calculs d'indices agronomiques.

\subsection{Données produites par Floreallndices}

\subsubsection{Une première description par les types de végétation dominants de la communauté : la typologie agronomique des prairies permanentes (ATOUS)}

Cette typologie des prairies permanentes a été mise au point dans le projet CASDAR ATOUS (Hulin et al., 2019). Elle a été utilisée pour établir des ponts entre trois typologies locales utilisées dans le projet: Massif central (Carrère et al., 2012), Alpes du Nord (Theau et al., 2018) et Pyrénées atlantiques (Arranz et al., 2016). Elle ne vise pas à se substituer aux typologies locales, mais nous paraît tout à fait légitime de par sa généricité pour être utilisée dans n'importe quelle situation en France métropolitaine. Elle permet, sur la base du relevé (phytosociologique notamment), de se représenter la physionomie de la prairie, en mobilisant principalement les grandes familles de plantes qui dominent dans la station et que nous appelons des types de végétation.

Les types de végétation sont obtenus par un cumul des recouvrements des 5 formes de vie (FDV) décrites en colonne $\mathrm{F}$ de FlorealData. Le tableau 3 en donne les règles de construction et leur fréquence pour 435 prairies de MidiPyrénées, qui n'ont pas été retournées depuis plus de 20 ans dans leur grande majorité.

Ces types de végétation correspondent au premier regard que l'agronome peut avoir lorsqu'il rentre dans la prairie. Si une espèce non graminéenne domine, elle est le signe d'un risque possible de perte de diversité ainsi qu'un possible signal d'alerte invasif(Dom) à considérer selon l'espèce et ses qualités fourragères (grandes ombellifères, renoncules, pissenlit...). Pour le regroupement des Cypéracées et des Juncacées (Cyju), dans notre cas d'étude, il s'agit de situations annonciatrices de milieux humides. Ces deux premiers types de végétation (Dom et $\mathrm{CyJu}$ ) représentent $15 \%$ des situations dans notre dispositif et sont assez corrélées avec une faible richesse spécifique. Le troisième type de végétation est assez rare et est constitué des stations où les graminées sont très dominantes (Gram). Pour l'agronome, il peut être annonciateur d'une faible diversité spécifique et, dans les milieux riches en nutriments, être la conséquence d'une intensification de la conduite fourragère. Le quatrième type de végétation est celui où les légumineuses (Leg) occupent au moins 20\% du recouvrement. Il est suffisamment original en agronomie pour être relevé (double du recouvrement moyen de la prairie permanente en France), car ces végétations sont remarquables de par leurs qualités fourragères, leur indépendance vis-à-vis de la fourniture en azote du sol et leur richesse spécifique. Enfin, le dernier type de végétation (Div) est par construction celui qui reste et qui possède les plus fortes richesses spécifiques avec le groupe des légumineuses.

Si cette première photographie de la parcelle donne un aperçu assez général de la physionomie de la végétation, elle nécessite, du fait d'une forte variabilité intratype, une analyse plus fine au travers du calcul des indices agroécologiques (Tab. 4).

\subsubsection{Des indices agroécologiques pour décrire les stations}

\subsubsection{Indices écologiques}

La richesse spécifique est le nombre d'espèces présentes dans la station (recouvrement relatif supérieur à 0 ).

Le CSI(Index de spécialisation de la communauté) est la moyenne arithmétique des SSI (Index de spécialisation des espèces) des espèces présentes dans la station. Créé pour la faune (Julliard et al., 2006), validé pour la flore (Turcati, 2011), il a été adapté pour qualifier les communautés d'un jeu de données phytosociologiques (Prud'homme et Theau, 2017). Des valeurs élevées de CSI indiquent une communauté dont les espèces sont à la fois fidèles et exclusives à la communauté, contrairement aux espèces généralistes. Le SSI est donné pour chaque espèce dans la colonne $\mathrm{D}$ de FlorealData.

$$
\frac{1}{n} \sum_{i=1}^{n} x_{i}
$$

où $\mathrm{x}_{\mathrm{i}}$ correspond au SSI des $\mathrm{n}$ espèces présentes.

Le CSI sans annuelles est un calcul identique mais excluant les espèces annuelles renseignées dans la colonne $\mathrm{E}$ de FlorealData par Thérophyte. Ces espèces annuelles sont souvent la résultante d'une perturbation agronomique ou écologique (en particulier les espèces rudérales). Elles peuvent marquer fortement un milieu sans pour autant avoir un lien direct avec la communauté prairiale vivace et ainsi augmenter artificiellement le CSI d'une parcelle si elles ne sont pas retirées du calcul. Cet indice porterait un sens différent dans les prairies du domaine méditerranéen, naturellement riches en thérophytes.

IndAn est le taux d'annuelles de la communauté (colonne E de FlorealData) et correspond à l'abondance des thérophytes. Le taux d'annuelles peut être, au sein des prairies permanentes de notre territoire, un signe de dégradation de la communauté.

$$
\left(\sum_{i=1}^{n} x_{i}\right) * 100
$$

où $\mathrm{x}_{\mathrm{i}}$ correspond à l'abondance des $\mathrm{n}$ espèces thérophytes de la station. 
Tableau 2. Liste des 43 types fonctionnels de plantes par types de végétation et indicateurs retenus pour le calcul des indices agronomiques. Table 2. List of 43 functional types of plants by type of vegetation and indicators used for the calculation of agronomic indices.

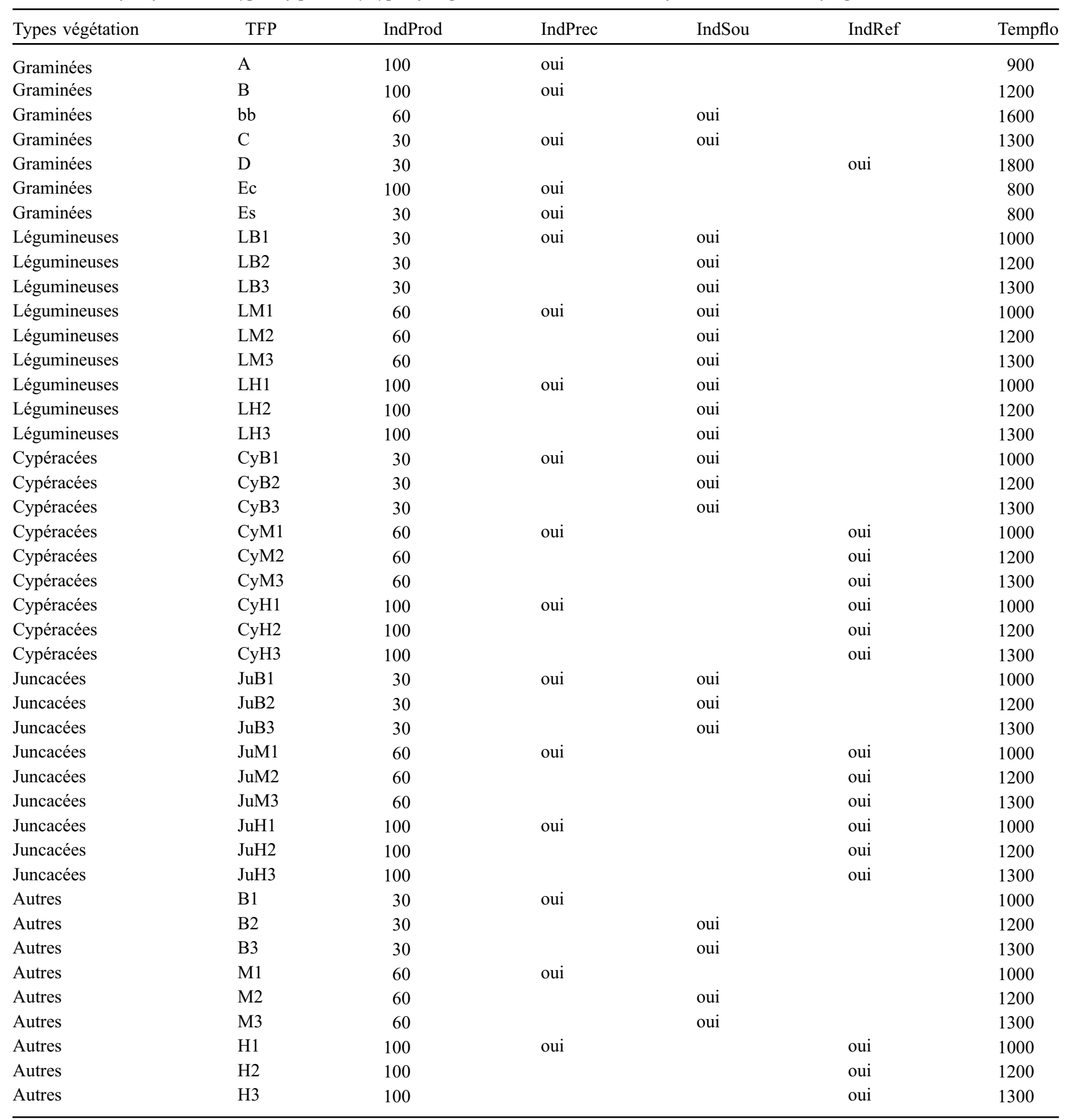

NbCoul est un comptage du nombre de couleurs potentielles différentes du vert, permises par la communauté, indépendamment de leur abondance. Il s'appuie sur la colonne $\mathrm{C}$ de FlorealData qui décrit la couleur dominante des fleurs.
RarCoul est un indice de rareté des couleurs permise par la communauté. L'abondance de chaque couleur est pondérée par un indice donné en tableau 5, qui est croissant avec sa rareté à Midi-Pyrénées. Il est propre à Midi-Pyrénées et s'appuie sur 
Tableau 3. Définition des types de végétation ATOUS, fréquence dans la base de données Midi-Pyrénées et liens avec la richesse spécifique. Les lettres différentes indiquent des différences statistiques de richesse spécifique entre types de végétation.

Table 3. Definition of ATOUS vegetation types, frequency in the Midi-Pyrénées database and links with specific richness. The different letters indicate statistical differences in species richness between types of vegetation.

\begin{tabular}{llcl}
\hline Type & \multicolumn{1}{c}{ Définition de la communauté } & Fréquence en Midi-Pyrénées (\%) & Richesse spécifique \\
\hline Dom & L'espèce non graminéenne $>20 \%$ de recouvrement relatif & 4 & $17^{\mathrm{a}}$ \\
CyJu & Cypéracée et Juncacée $>20 \%$ d'abondance & 11 & $23^{\mathrm{a}}$ \\
Gram & Abondance des graminées $>75 \%$ & 1 & $27^{\mathrm{ab}}$ \\
Leg & Abondance des Légumineuses $>20 \%$ & 8 & $33^{\mathrm{b}}$ \\
Div & Tout ce qui n'est pas retenu ci-dessus & 75 & $33^{\mathrm{b}}$ \\
\hline
\end{tabular}

Tableau 4. Liste des indices agroécologiques calculés dans FlorealIndices.

Table 4. List of agroecological indices calculated in FlorealIndices.

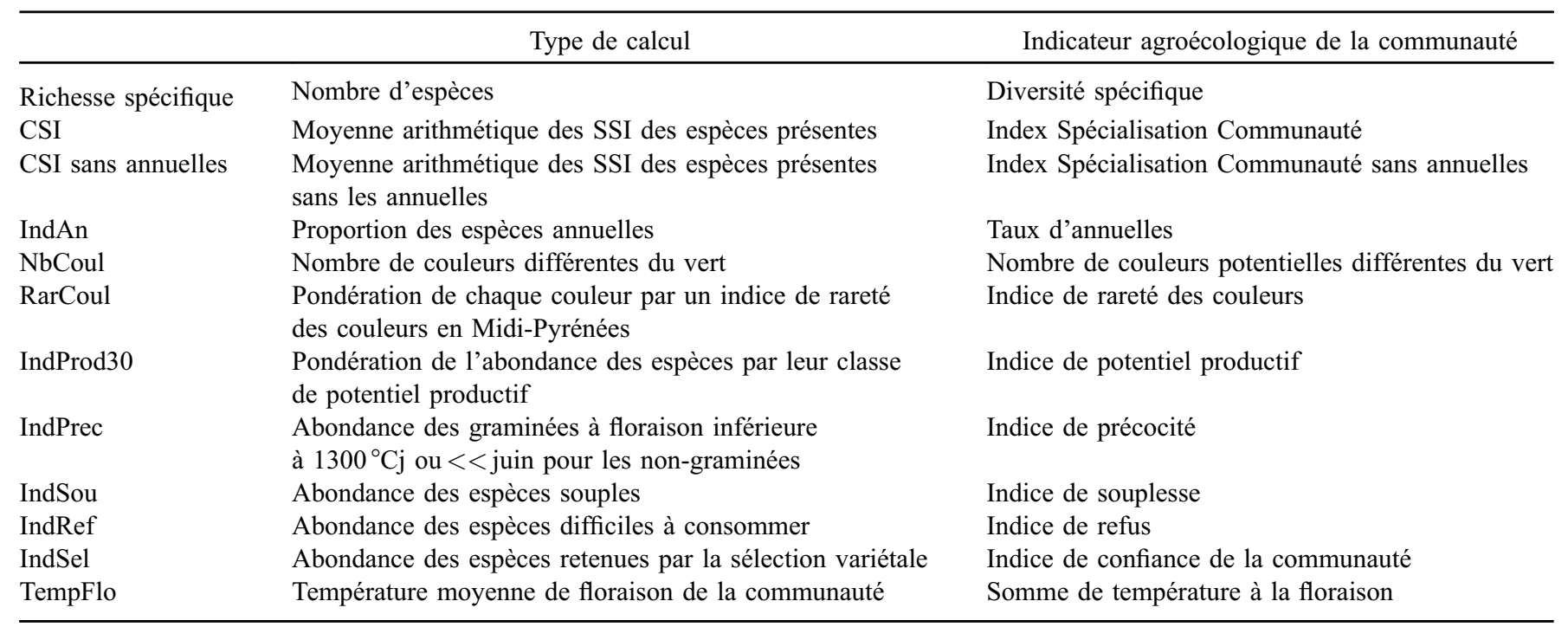

une fréquence de chaque couleur des espèces au sein de la région.

$$
\begin{gathered}
\left(\sum_{i=1}^{n} b_{i}\right) * 1+\left(\sum_{i=1}^{m} j_{i}\right) * 1+\left(\sum_{i=1}^{k} r_{i}\right) * 2 \\
+\ldots+\left(\sum_{i=1}^{s} p_{i}\right) * 6
\end{gathered}
$$

où

- $b_{i}$ correspond à l'abondance des $n$ espèces blanches ;

- $\mathrm{j}_{\mathrm{i}}$ correspond à l'abondance des $\mathrm{m}$ espèces jaunes ;

- $\mathrm{r}_{\mathrm{i}}$ correspond à l'abondance des $\mathrm{k}$ espèces roses;

- $\mathrm{p}_{\mathrm{i}}$ correspond à l'abondance des $s$ espèces pourpres.

\subsubsection{Indices agronomiques}

Quatre de ces indices agronomiques (IndPro, IndPrec, IndSou et IndRef) ont été initialement décrits (Theau et al., 2019). Le tableau 2 précise les modalités d'affectation de chaque type fonctionnel de plantes aux indices agronomiques.
IndProd30 exprime le potentiel de production permis par la communauté. Il combine des connaissances issues d'une approche fonctionnelle pour les graminées (Cruz et al., 2010 ; Duru et al., 2010) et pour les espèces non graminéennes (Theau et al., 2017). L'indice cumule l'abondance de chaque type fonctionnel de plante, pondéré par un indice de productivité de 100, 60 ou 30 (Tab. 2). IndProd30 évolue donc entre 30 et 100 .

$$
\sum_{i=1}^{n} x_{i}+\sum_{i=1}^{p} y_{i}+\sum_{i=1}^{k} z_{i}
$$

où

- $\mathrm{x}_{\mathrm{i}}$ correspond à l'abondance des $\mathrm{n}$ espèces de TFP 100 ;

- $\mathrm{y}_{\mathrm{i}}$ correspond à l'abondance des $\mathrm{p}$ espèces de TFP 60 ;

- $\mathrm{z}_{\mathrm{i}}$ correspond à l'abondance des $\mathrm{k}$ espèces de TFP 30.

L'indice de production n'a pas la prétention d'estimer une biomasse aérienne, mais permet de classer les prairies d'un même milieu entre elles. Pour approcher le potentiel de production de biomasse aérienne, il est possible de pondérer 
Tableau 5. Fréquence des couleurs sur 768 espèces prairiales de Midi-Pyrénées et affectation d'un coefficient de rareté de la couleur. Table 5. Color frequency on 768 meadow species in the MidiPyrénées and assignment of a color rarity coefficient.

\begin{tabular}{lll}
\hline $\begin{array}{c}\text { Couleur } \\
\text { dominante des fleurs }\end{array}$ & $\begin{array}{c}\text { Fréquence } \\
\text { couleur }(\%)\end{array}$ & $\begin{array}{c}\text { Coefficient } \\
\text { couleur }\end{array}$ \\
\hline Blanc & 31 & 1 \\
Jaune & 26 & 1 \\
Rose & 17 & 2 \\
Bleu & 12 & 2 \\
Marron, brun, noir & 6 & 3 \\
Violet & 5 & 4 \\
Rouge & 2 & 5 \\
Pourpre & 1 & 6 \\
\hline
\end{tabular}

son indice de production par un potentiel en base 100 obtenu par avis d'expert pour un territoire donné (Duru et al., 2010).

IndPrec est un indice de précocité qui cumule l'abondance des espèces dont la floraison est inférieure ou égale à un cumul des sommes de températures de $1300^{\circ} \mathrm{C}$ jours, ou inférieur ou égal au mois de mai pour les espèces non graminéennes (Tab. 2). Le complément pour arriver à l'abondance totale de la station représente la tardiveté.

$$
\left(\sum_{i=1}^{n} x_{i}\right) * 100
$$

où $\mathrm{x}_{\mathrm{i}}$ correspond à l'abondance des $\mathrm{n}$ espèces précoces.

Dans les lignes 965, 966 et 967, il est fait un codage plus précis de la station, en trois classes de précocité de la floraison. Il permet d'avoir, en base 100, trois compartiments de précocité de floraison de la station qui décrivent plus finement sa dynamique dans le temps :

- \% précoce $=$ graminées $<1000^{\circ} \mathrm{Cj}$ ou non-graminées $<<$ mai ;

- \% interm $=$ graminées de 1000 à $1300^{\circ} \mathrm{Cj}$ ou non-graminées $=$ mai

- \% tardif $=$ graminées $>1300{ }^{\circ} \mathrm{Cj}$ ou non-graminées $>>$ mai.

IndSou est un indicateur de souplesse. La souplesse d'utilisation d'une prairie est sa capacité à conserver une caractéristique stable (productivité, digestibilité de l'herbe...) sans dégradation de celle-ci sur un laps de temps long. Les prairies souples vont se caractériser par une bonne persistance du rapport feuille/tige de la communauté. Celui-ci peut être lié à certaines espèces (types fonctionnels de graminées $\mathrm{B}$ et $\mathrm{C}$, légumineuses), ainsi qu'à l'apparition d'espèces assez tardives à tardives dans la végétation, qui viennent renouveler l'abondance de feuilles dans la communauté. Le caractère souple est donné pour chaque TFP dans le tableau 2.

$$
\left(\sum_{i=1}^{n} x_{i}\right) * 100
$$

où $\mathrm{x}_{\mathrm{i}}$ correspond à l'abondance des $\mathrm{n}$ espèces souples.

IndRef est un indicateur qui permet de quantifier la part des espèces potentiellement difficiles à consommer par les animaux. Il s'agit des graminées de type fonctionnel D qui lignifient rapidement et des espèces non graminéennes de grande taille $(>90 \mathrm{~cm})$, qui fabriquent une tige de grosse section. Celle-ci est généralement refusée au pâturage ou génère des contraintes de séchage (pourrissement) dans les fourrages récoltés. Ces espèces peuvent être présentes dans les prairies, mais au-delà d'une certaine abondance, elles génèrent des contraintes d'usage, nécessitant une utilisation généralement plus précoce pour être acceptées ou une valorisation par des animaux peu exigeants sur le plan de l'alimentation. En fourrages conservés, les tiges difficiles à sécher au champ nécessitent un temps de dessiccation supérieur aux feuilles. Cela nécessite des opérations mécaniques de retournement des foins plus nombreuses, qui conduisent à une brisure des feuilles et à une diminution de la valeur alimentaire des fourrages. Le caractère refus est donné pour chaque TFP dans le tableau 2.

$$
\left(\sum_{i=1}^{n} x_{i}\right) * 100
$$

où $\mathrm{x}_{\mathrm{i}}$ correspond à l'abondance des $\mathrm{n}$ espèces facilement refusées.

IndSel est un indicateur destiné aux éleveurs qui se questionnent sur le retournement de leur prairie permanente. Il permet de quantifier, dans la station, la part des végétations utilisée par la sélection variétale (colonne $\mathrm{K}$ de FlorealData = oui). Nous le considérons comme un indicateur de confiance dans les espèces présentes dans la communauté. Sur un échantillon de 435 prairies permanentes de Midi-Pyrénées, sa valeur moyenne est de $36 \%$, avec des valeurs extrêmes allant de 0 à $79 \%$ de l'abondance de la communauté.

$$
\left(\sum_{i=1}^{n} x_{i}\right) * 100
$$

où $\mathrm{x}_{\mathrm{i}}$ correspond à l'abondance des $\mathrm{n}$ espèces ou genres inscrits en sélection variétale.

TempFlo est une estimation de la température moyenne de floraison de la communauté. La température moyenne de floraison donnée pour chaque TFP dans le tableau 2 est pondérée par l'abondance de chaque TFP. Cet indicateur permet donc de situer un état phénologique moyen de la parcelle dans le temps, en regard d'une date de fauche ou de pâturage déclarée par un éleveur.

$$
\sum_{i=1}^{a} t_{i}+\sum_{i=1}^{b} u_{i}+\sum_{i=1}^{c} v_{i}+\sum_{i=1}^{d} w_{i}+\sum_{i=1}^{e} x_{i}+\sum_{i=1}^{f} y_{i}+\sum_{i=1}^{g} z_{i}
$$

où

- $t_{i}$ correspond à l'abondance des $a$ espèces à floraison $800^{\circ} \mathrm{Cj}$;

- $u_{i}$ correspond à l'abondance des $b$ espèces à floraison $900{ }^{\circ} \mathrm{Cj}$; 
Tableau 6. Comparaison des moyennes de chaque indice pour deux des dispositifs (Prairies fleuries et MAE) en Midi-Pyrénées. Analyse de variance et test de comparaison de moyenne (Bonferroni). Des lettres différentes entre dispositifs indiquent des moyennes statistiquement différentes $(p<0,05)$.

Table. Comparison of the means of each index for two of the devices (Prairies fleuries and MAE) in Midi-Pyrénées. Analysis of variance and mean comparison test (Bonferroni). Different letters between devices indicate statistically different means $(\mathrm{p}<0.05)$.

\begin{tabular}{lccc}
\hline 435 Prairies de Midi-Pyrénées & Moyenne Midi-Pyrénées & Moyenne Prairies Fleuries & Moyenne MAE \\
\hline Richesse spécifique & 31 & $36^{\mathrm{b}}$ & $27^{\mathrm{a}}$ \\
IndAn & 7 & $11^{\mathrm{b}}$ & $4^{\mathrm{a}}$ \\
CSI sans annuelles & 1,7 & $7^{\mathrm{a}}$ & $1,9^{\mathrm{b}}$ \\
NbCoul & 7 & $19^{\mathrm{a}}$ & $30^{\mathrm{b}}$ \\
RarCoul & 23 & $62^{\mathrm{b}}$ & $60^{\mathrm{a}}$ \\
IndProd & 63 & $77^{\mathrm{b}}$ & $46^{\mathrm{a}}$ \\
IndPrec & 66 & $56^{\mathrm{b}}$ & $39^{\mathrm{a}}$ \\
\% précoce & 51 & $23^{\mathrm{b}}$ & $18^{\mathrm{a}}$ \\
\% interm & 20 & $20^{\mathrm{a}}$ & $43^{\mathrm{b}}$ \\
In Tardif & 28 & $41^{\mathrm{ns}}$ & $40^{\mathrm{ns}}$ \\
IndRef & 39 & $4^{\mathrm{a}}$ & $29^{\mathrm{b}}$ \\
IndSel & 13 & $1098^{\mathrm{a}}$ & $18^{\mathrm{a}}$ \\
TempFlo & 36 & $4^{\mathrm{b}}$ & \\
\hline
\end{tabular}

- $v_{i}$ correspond à l'abondance des $c$ espèces à floraison $1000^{\circ} \mathrm{Cj}$;

- $w_{i}$ correspond à l'abondance des $d$ espèces à floraison $1200^{\circ} \mathrm{Cj}$

$-x_{i}$ correspond à l'abondance des $e$ espèces à floraison $1300^{\circ} \mathrm{Cj}$;

- $y_{i}$ correspond à l'abondance des $f$ espèces à floraison $1600^{\circ} \mathrm{Cj}$;

$-z_{i}$ correspond à l'abondance des $g$ espèces à floraison $1800^{\circ} \mathrm{Cj}$.

Il est possible de construire des abaques de transformation des dates calendaires en cumul des températures moyennes journalières sur tout le territoire français grâce à l'application suivante: https://www6.toulouse.inrae.fr/agir/Les-equipes/ MAGELLAN/Realisations/Herb-age.

TempFlo est une information essentielle, tant pour l'agronome qui s'intéresse à la qualité des fourrages, que pour le botaniste écologue qui se questionne sur l'impact des pratiques sur la dynamique des végétations ou pour l'entomologiste sur les liens entre flore et insectes (Prud'homme et Theau, 2017).

Ces indices sont visibles pour chaque station à partir des cellules M786 à M800.

\section{Application sur une base de données de prairies permanentes de Midi-Pyrénées}

\subsection{Comparaisons de deux dispositifs: Concours Pratiques agroécologiques et Mesures agroenvironnementales (MAE)}

Les parcelles soumises au concours «Pratiques agroécologiques : prairies et parcours » ou inscrites en MAE «Prairies humides » ont des particularités qui devraient ressortir au travers de nos indicateurs. Les premières ont pour objectif, afin d'être primées, de présenter un compromis entre les caractéristiques agronomiques et la biodiversité, alors que les secondes ont pour point commun d'appartenir toutes à des milieux humides.

Ces deux dispositifs regroupent respectivement 221 et 143 stations. Les indices permettent de comparer ces dispositifs. Pour cela, nous avons utilisé une analyse de variance (Tab. 6).

Les prairies soumises au concours «Pratiques agroécologiques: prairies et parcours » présentent des indices de productivité, de précocité, d'abondance des annuelles et de richesse spécifique significativement supérieurs aux MAE. Bien que la richesse spécifique des prairies en MAE soit plus faible, celles-ci présentent, sur le plan environnemental, des niveaux de CSI et de rareté des couleurs qui sont significativement plus forts. Ces deux indices soulignent l'intérêt environnemental des mesures MAE, alors que leur potentiel élevé de refus met en avant des contraintes d'usage agronomique nécessitant des compensations économiques légitimes si l'on veut préserver ces habitats.

\subsection{Distribution des prairies de Midi-Pyrénées pour chacun des indices}

Pour illustrer la gamme des valeurs observées pour chacun des indices, nous présentons leurs histogrammes de fréquences pour 435 prairies permanentes de Midi-Pyrénées. La forte amplitude de ces distributions retrace les conditions stationnelles contrastées de Midi-Pyrénées (altitude, sol, climat, usage...). Il ne s'agit pas ici de discuter ces résultats, mais de visualiser la gamme de valeurs potentielles de chaque variable sur un large échantillon de prairies permanentes. La figure 1 peut ainsi être utilisée par les utilisateurs potentiels de 
FlorealIndices afin de positionner leurs résultats et d'aider à leur interprétation.

\section{Discussions et conclusion}

La feuille de calcul FlorealIndices permet de passer du seul relevé phytosociologique à une description agronomique et écologique de la prairie. Elle est destinée à tous les conseillers et gestionnaires qui sauront s'armer de relevés botaniques par compétence ou par collaboration avec un botaniste. Elle permet de valoriser la base de données FlorealData élaborée par le CBNPMP et l'INRAE. Sa mise en œuvre permet d'ouvrir le dialogue avec l'éleveur, en objectivant des critères qu'il utilise dans la gestion quotidienne des prairies, tels que la productivité, la précocité, la souplesse d'exploitation, la qualité de l'herbe au travers de sa phénologie, tout en intégrant une dimension environnementale. Ces éléments sont également utiles aux animateurs de politiques de protection de la nature, permettant de partager des connaissances sur l'écologie de la prairie et d'envisager des actions conservatoires mieux intégrées à la gestion agricole.

Aujourd'hui, le paramétrage du fichier est suffisant pour être importé dans un module de terrain qui, dès la sortie de la parcelle, permettrait de rendre compte des caractéristiques de la prairie auprès de l'éleveur: (i) une description de la végétation en type de prairie permanente; (ii) une description de la communauté grâce aux indicateurs agronomiques et écologiques.

L'automatisation de ce relevé pourrait permettre de dépasser la seule échelle parcellaire, qui est insuffisante pour avoir un raisonnement cohérent du système herbager (Duru et al., 2011; Farruggia et al., 2012), et de caractériser un ensemble de parcelles ayant des fonctions similaires dans le système (ensemble de la sole fauchée, ou de la sole pâturée par le lot en production, ou les génisses...). Un botaniste professionnel peut réaliser une dizaine de relevés phytosociologiques dans la journée, ce qui permet d'instruire un nombre de parcelles cohérent avec la dimension de la sole. L'ensemble de ces informations serait donc utile pour construire un discours partagé entre agronomes et écologues à un niveau d'échelle pertinent pour l'éleveur dans sa gestion. Elles permettraient d'identifier les compromis possibles entre parcelles, afin de concevoir une gestion agroécologique des prairies en cohérence avec le projet de production de l'éleveur, ou à une plus grande échelle de décrire des paysages, des zones biogéographiques ou des territoires, comme cela a été réalisé dans le programme CASDAR ATOUS. Un travail en cours en Aubrac devrait permettre d'évaluer la complémentarité entre cette approche d'indices calculés sur la base du relevé phytosociologique et celle proposée par les typologies multifonctionnelles comme celle du Massif central (Galliot et al., 2019).

Remerciements. Ce projet a été financé par le FEDER Massif central (MC0019617) et la région Occitanie «Mieux comprendre et valoriser les prairies naturelles pour leur donner une place de choix dans les exploitations du HautLanguedoc», piloté par le Parc naturel régional du HautLanguedoc.

Nous remercions tout particulièrement Marie Julie Parayre et Michel Castan (Parc naturel régional du Haut-Languedoc) pour leur accompagnement technique, leur enthousiasme et leur confiance.

Cette publication est également l'aboutissement de travaux collaboratifs menés avec les gestionnaires d'espace naturels depuis de longues années. Nous remercions particulièrement Jean-Guillaume Thiébault (Parc national des Pyrénées), Carine Chatain et Béatrice Loncan (Chambre d'agriculture des Hautes-Pyrénées), Claire Lemouzy (ADASEA du Gers), Julien Aït El Mekki et Gaëlle Fédrigo (Parc naturel régional des Pyrénées ariégeoises), Camille Favier et Marie-Julie Parayre (PNR Haut-Languedoc), Laure Jacob (PNR Grands Causses), Benoît Delmas (Chambre d'agriculture de l'Aveyron), Nicolas Cayssiol et Philippe Bernié (ADASEA d'Oc), Emmanuel Campagne et Jean-Bernard Mis (Chambre d'agriculture du Tarn).

Ont contribué aussi, d'une façon indirecte mais fondamentale, tous les membres de jury de concours prairies fleuries et tous les agriculteurs (près de deux cents pour cette base de données !) Merci à tous.

Enfin, merci aux équipes du Conservatoire botanique national des Pyrénées et de Midi-Pyrénées et de l'INRAE de Toulouse pour leur accompagnement.

\section{Références}

Arranz JM, Mareaux M, Inarra P, Bernos N, Olha E, Gascouat P et al. 2016. Prairies permanentes basco-béarnaises: Une typologie pour évaluer les services écosystémiques rendus par les prairies et par les exploitations. Fourrages 228: 233-241. https://afpf-asso.fr/index. php? secured_download $=2193 \&$ token $=\mathrm{c} 16 \mathrm{~b} 0 \mathrm{ba} 6 \mathrm{e} 27 \mathrm{c} 1 \mathrm{efb} 0$ d74a94026afe 433 .

Braun-Blanquet J. 1964. Pflanzensoziologie. Grundzüge der vegetationskunde, $3^{\mathrm{e}}$ éd. Wien (Austria), New York (USA): Springer, $865 \mathrm{p}$.

Carrère P, Seytre L, Piquet M, Landrieaux J, Rivière J, Chabalier C et al. 2012. Une typologie multifonctionnelle des prairies des systèmes laitiers AOP du Massif central combinant des approches agronomiques et écologiques. Fourrages 209: 9-21. https://afpfasso.fr/index.php?secured_download $=1964 \&$ token $=$ c16b0ba6e 27 c1efb0d74a94026afe433.

Cruz P, Theau JP, Lecloux E, Jouany C, Duru M. 2010. Typologie fonctionnelle de graminées fourragères pérennes: une classification multitraits. Fourrages 201: 11-17. https://afpf-asso.fr/index. php? secured_download $=1880 \&$ token $=\mathrm{c} 16 \mathrm{~b} 0 \mathrm{ba} 6 \mathrm{e} 27 \mathrm{c} 1 \mathrm{efb} 0$ d74a94026afe 433 .

Data INRAE. [2019/09/23]. https://data.inrae.fr/.

Dufrêne M. 1998. Cours de Phytosociologie. Partie «analyse de données». version $\beta$. 1-1/12/98. Disponible sur: http://www.biol. ucl.ac.be/ecol/cours/phytosocio/home.html.

Duru M, Cruz P, Jouany C, Theau JP. 2010. Herb'type $\odot$ : un nouvel outil pour évaluer les services de production fournis par les prairies permanentes. INRA Productions Animales 23(4): 319-332. https:// doi.org/10.20870/productions-animales.2010.23.4.3311.

Duru M, Theau JP, Hossard L, Martin G, Cruz P. 2011. Diversité de la composition fonctionnelle de la végétation au sein d'une prairie et entre prairies : caractérisation et analyse dans des élevages herbagers. Fourrages 2011: 61-73. https://hal.inrae.fr/hal-02650578.

Farruggia A, Lacour C, Zapata J, Piquet M, Baumont B, Carrère P et al. 2012. DIAM, un diagnostic innovant déclinant les équilibres, production, environnement et qualité des fromages au sein des systèmes fourragers des zones AOP du Massif central. Rencontres Recherches Ruminants 19: 13-16. 
Galliot JN, Hulin S, Bonsacquet E, Carrère P. 2019. Apprécier les compromis entre services à travers la typologie multifonctionnelle des prairies du Massif central. Fourrages 237: 67-74. https://afpfasso.fr/index.php?secured_download=3418\&token= 85254985d2a97e39944b71f2af2a1b25.

Hulin S, Farruggia A, Theau JP, Cayre P, Chauvat S, Pauthenet Yet al. 2019. ATOUS - Vers une approche territoriale de l'autonomie fourragère et des services rendus par les systèmes fourragers à dominante herbagère en production fromagère AOP de montagne. Innovations Agronomiques 71: 95-108. https://doi.org/10.15454/ ynaxsb.

Julliard R, Clavel J, Devictor V, Jiguet F, Couvet D. 2006. Spatial segregation of specialists and generalists in bird communities. Ecology Letters 9: 1237-1244. https://doi.org/10.1111/ j.1461-0248.2006.00977.x.

Plantureux S, Thirion G, Georgel R, Bellicam MJ, Parthonneau JL, Henry JM. 2012. La typologie des prairies du massif vosgien: Un outil pour évaluer l'autonomie fourragère. Journées AFPF. Les atouts des prairies permanents pour demain, 3-4 avril 2012. https:/www.parc-vosges-nord.fr/wp-content/uploads/2018/11/ typologie-des-prairies-permanentes-des-ballons-des-vosges-2006. pdf.

Prud'homme F, Theau JP. 2017. Phytosociologie et agronomie à la rencontre des prairies fleuries. In: Actes des Rencontres naturalistes de Midi-Pyrénées, Auch, 12 et 13 février 2016. pp. 69-73. https://hal.archives-ouvertes.fr/hal-01607765.

Prud'homme F, Theau JP. 2019. La phytosociologie au service de l'agroécologie : L'exemple des prairies humides de Midi-Pyrénées. Actes du colloque international de phytosociologie de Bailleul: Valeurs et usages des zones humides. 26-30 septembre 2017.
Documents phytosociologiques 12: 285-299. https://hal.inrae.fr/ hal-02622296.

Theau JP, Choisis JP. 2016. Prairie permanente: Définition. Dictionnaire d'Agroecologie. https://dicoagroecologie.fr/encyclo pedie/prairie-permanente/.

Theau J-P, Prud'homme F. 2021. FlorealData: Des traits de vie d'espèces végétales pour le calcul d'indices agronomiques et écologiques des communautés prairiales. Cah Agric Vol: 210051. https://doi.org/10.1051/cagri/2021022.

Theau JP, Pauthenet Y, Cruz P. 2017. Une typologie des espèces non graminéennes pour mieux caractériser la diversité et la valeur d'usage des prairies permanentes. Fourrages 232: 321-329. https:// afpf-asso.fr/index.php? secured_download $=2240 \&$ token $=$ c16b0ba6e27c1efb0d74a94026afe433.

Theau JP, Malvoisin T, Faugeroux F, Pauthenet Y. 2018. Dialog'Alpes: Un outil pour valoriser la diversité des prairies permanentes dans les exploitations d'élevage bovin laitier. Fourrages 234: 131142. https://afpf-asso.fr/index.php?secured_download= 2263\&token $=\mathrm{c} 16 \mathrm{~b} 0 \mathrm{ba6e} 27 \mathrm{c} 1 \mathrm{efb} 0 \mathrm{~d} 74 \mathrm{a} 94026 \mathrm{afe} 433$.

Theau JP, Prud'homme F, San Cristobal M, Sirami C. 2019. Complémentarités entre approches écologique et agronomique pour une gestion agroécologique des prairies permanentes en MidiPyrénées. Fourrages 237: 5-14. https://afpf-asso.fr/revue/agrono mie-et-ecologie-un-duo-gagnant-pour-comprendre-et-gerer-lesprairies? $\mathrm{a}=2192$.

Turcati L. 2011. Mesurer la biodiversité pour comprendre l'effet des perturbations sur les communautés végétales: apport des caractéristiques écologiques et évolutives des espèces. Museum national d'histoire naturelle (Paris): UPMC Sorbonne Université, 264 p. https://www.vigienature.fr/sites/vigienature/files/atoms/files/ vf_these_laure_turcati_1.pdf.

Citation de l'article : Theau J-P, Prud'homme F. 2021. FlorealIndices : Une feuille de calcul pour produire des indices agroécologiques de prairies permanentes à partir du relevé de végétation. Cah. Agric. 30: 37. 